\title{
A CHARACTERIZATION OF THE GENERALIZED VERONESE SURFACES
}

\author{
TAKEHIRO ITOH
}

(Communicated by David G. Ebin)

\begin{abstract}
We prove that compact $m$-regular minimal surfaces in a sphere are generalized Veronese surfaces if the Gaussian curvature satisfies an inequality.
\end{abstract}

The Gaussian curvature of a surface is an intrinsic value. It is well known that minimal surfaces of constant curvature in a sphere are rigid [1]. These surfaces are called generalized Veronese surfaces in [3 or 5]. Now, we may conjecture that generalized Veronese surfaces can be characterized only by the Gaussian curvature.

In this paper, we will prove that this conjecture is true for $m$-regular minimal surfaces ( $m$-regular means that the $k$ th normal space $N_{k}$ satisfies $\operatorname{dim} N_{k}>\operatorname{dim} N_{k-1}$ for $k=1,2, \ldots, m$, where $\operatorname{dim} N_{0}=0$, and 0-regular mapping means an ordinary regular one). That is, we prove the following:

THEOREM. Let $M$ be a compact connected oriented surface minimally immersed in a unit sphere through the $m$-regular immersion. If its Gaussian curvature $K$ satisfies

$$
\frac{2}{(m+2)(m+3)} \leq K \leq \frac{2}{(m+1)(m+2)}, \quad 0 \leq m
$$

then $M$ is a generalized Veronese surface.

1. Preliminaries. Let $\tilde{M}$ be a $(2+\nu)$-dimensional Riemannian manifold of constant curvature $\tilde{c}$, and $M$ a 2-dimensional Riemannian manifold which is immersed in $\tilde{M}$ by the immersion $x: M \rightarrow \tilde{M}$. Let $\left(e_{1}, e_{2}, \ldots, e_{2+\nu}\right)$ be an orthonormal frame field over $\tilde{M}$ such that $\left(e_{1}, e_{2}\right)$ is an orthonormal frame field over $M$. Let $\omega_{A}$ and $\omega_{A B}$ be basic and connection forms with respect to the above orthonormal frames. Then, as is well known, we have

$$
\begin{gathered}
\omega_{\alpha}=0, \quad \omega_{i \alpha}=\sum_{j} h_{i j}^{\alpha} \omega_{j}, \quad h_{i j}^{\alpha}=h_{j i}^{\alpha}, \\
d \omega_{i}=\omega_{i j} \wedge \omega_{j}, \quad i \neq j \\
d \omega_{i j}=\sum_{k} \omega_{i k} \wedge \omega_{k j}-\Omega_{i j}, \quad \Omega_{i j}=\frac{1}{2} \sum_{k, l} R_{i j k l} \omega_{k} \wedge \omega_{l}, \\
R_{i j k l}=\tilde{c}\left(\delta_{i k} \delta_{j l}-\delta_{i l} \delta_{j k}\right)+\sum_{\alpha}\left(h_{i k}^{\alpha} h_{j l}^{\alpha}-h_{i l}^{\alpha} h_{j k}^{\alpha}\right)
\end{gathered}
$$

Received by the editors November 20, 1987.

1980 Mathematics Subject Classification (1985 Revision). Primary 53A10; Secondary 53C42.

Key words and phrases. $m$-regular immersion, generalized Veronese surfaces. 


$$
\begin{gathered}
d \omega_{\alpha \beta}=\sum_{\gamma} \omega_{\alpha \gamma} \wedge \omega_{\gamma \beta}-\Omega_{\alpha \beta}, \quad \Omega_{\alpha \beta}=\frac{1}{2} \sum_{i, j} R_{\alpha \beta i j} \omega_{i} \wedge \omega_{j} \\
R_{\alpha \beta i j}=\sum_{k}\left(h_{i k}^{\alpha} h_{k j}^{\beta}-h_{j k}^{\alpha} h_{k i}^{\beta}\right)
\end{gathered}
$$

where we use the following convention on the ranges of indices:

$$
1 \leq A, B, \ldots \leq 2+\nu, \quad 1 \leq i, j, \ldots \leq 2, \quad 3 \leq \alpha, \beta, \ldots \leq 2+\nu .
$$

$M$ is said to be minimal if its mean curvature $\frac{1}{2} \sum_{i, \alpha} h_{i i}^{\alpha} e_{\alpha}$ vanishes identically, i.e., if trace $H_{\alpha}=0$ for all $\alpha, H_{\alpha}=\left(h_{i j}^{\alpha}\right)$.

Let $M_{p}$ be the tangent space at $p$ to $M$ and $N(p)$ the normal space of $M$ at $p$. We can write the (first) shape operator (the second fundamental form) $\varphi_{1}$ as

$$
\varphi_{1}(X, Y)=\sum h_{i j}^{\alpha} \omega_{i}(X) \omega_{j}(Y) e_{\alpha}, \quad X, Y \in M_{p}
$$

Now we define $h_{i_{1} \cdots i_{k}}, 2 \leq k$, by

$$
\sum h_{i_{1} \cdots i_{k} m}^{\alpha} \omega_{m}:=d h_{i_{1} \cdots i_{k}}^{\alpha}+\sum h_{i_{1} \cdots i_{j-1} m i_{j+1} \cdots i_{k}}^{\alpha} \omega_{m i_{j}}+\sum h_{i_{1} \cdots i_{k}}^{\beta} \omega_{\beta \alpha} .
$$

Then we can define the $k$ th shape operator (the $k$ th second fundamental form) $\varphi_{k}$ as the multilinear mapping from $M_{p} \times \cdots \times M_{p}$ into $N(p)$ by

$$
\varphi_{k}\left(X_{1}, X_{2}, \ldots, X_{k+1}\right):=\sum h_{i_{1} \cdots i_{k+1}}^{\alpha} \omega_{i_{1}}\left(X_{1}\right) \cdots \omega_{i_{k+1}}\left(X_{k+1}\right) e_{\alpha}
$$

where $X_{j} \in M_{p}, j=1,2, \ldots, k+1$. We define the $k$ th normal space $N_{k}(p)$ of $M$ at $p$ as

$$
N_{k}(p):=\operatorname{Span}\left\{\varphi_{1}\left(X_{1}, X_{2}\right), \varphi_{2}\left(X_{1}, X_{2}, X_{3}\right), \ldots, \varphi_{k}\left(X_{1}, \ldots, X_{k+1}\right)\right\},
$$

where $X_{j} \in M_{p}, j=1,2, \ldots, k+1$. The immersion is said to be $m$-regular if $\operatorname{dim} N_{k}(p)>\operatorname{dim} N_{k-1}(p)$ at each point $p \in M$ for all $k=1,2, \ldots, m$, where $\operatorname{dim} N_{0}(p)=0$. A 0-regular mapping means an ordinary one, so an immersion is 0 -regular.

2. Compact minimal positive curvature surfaces in a space form. Let $M$ be a compact connected oriented surface which is minimally immersed in a $(2+\nu)$ dimensional Riemannian manifold $\tilde{M}$ by the $m$-regular immersion. We suppose that the Gaussian curvature $K$ of $M$ is positive. Let $U$ be a neighborhood of a point $p \in M$ in which there exist isothermal coordinates $(u, v)$ and a frame field $\left(e_{1}, e_{2}\right)$ such that

$$
d s^{2}=E\left\{d u^{2}+d v^{2}\right\}, \quad \omega_{1}=\sqrt{E} d u, \quad \omega_{2}=\sqrt{E} d v,
$$

where $d s$ is the line element of $M$ and $E=E(u, v)$ is a positive function on $U$. Since $M$ is minimal in $U$, we may write

$$
\omega_{1 \alpha}=f_{\alpha} \omega_{1}+g_{\alpha} \omega_{2}, \quad \omega_{2 \alpha}=g_{\alpha} \omega_{1}-f_{\alpha} \omega_{2}, \quad 2<\alpha,
$$

where $f_{\alpha}$ and $g_{\alpha}$ are functions on $U$. Then, using the structure equations, we easily see that the complex valued function

$$
w_{1}(z, \bar{z})=E^{2}\left(\left|G_{1}\right|^{2}-\left|F_{1}\right|^{2}\right)+2 i E^{2}\left\langle G_{1}, F_{1}\right\rangle
$$


is holomorphic in $z=u+i v$, where $F_{1}=\sum f_{\alpha} e_{\alpha}$ and $G_{1}=\sum g_{\alpha} e_{\alpha}$. For a tangent vector $X=\cos \theta \cdot e_{1}+\sin \theta \cdot e_{2}$ of $M_{p}$, we have the mapping $\tilde{\varphi}_{1}$ from $M_{p}$ into $N(p)$ as follows:

$$
\tilde{\varphi}_{1}(X):=\varphi_{1}(X, X)=\cos 2 \theta \cdot F_{1}+\sin 2 \theta \cdot G_{1},
$$

where $F_{1}=\sum_{\alpha} f_{\alpha} e_{\alpha}=\sum_{\alpha} h_{11}^{\alpha} e_{\alpha}$ and $G_{1}=\sum g_{\alpha} e_{\alpha}=\sum h_{12}^{\alpha} e_{\alpha}$. Then we have

LEMMA 1. At each point $p$ of $M$, the image of the tangent unit circle $S_{p}^{1}$ under the mapping $\tilde{\varphi}_{1}$ is a point or a circle according as $\operatorname{dim} N_{1}(p)=0$ or $\neq 0$, where $S_{p}^{1}=\left\{X \in M_{p}|| X \mid=1\right\}$.

ProOF. We easily see that $\left|w_{1}(z, \bar{z})\right|^{2} / E^{4}$ is a differentiable function on $M$. Since $M$ is compact, $\left|w_{1}(z, \bar{z})\right|^{2} / E^{4}$ takes the maximum $A$ at some point $p_{0}$ of $M$. If $A>0$, then there exists a neighborhood $U$ of $p_{0}$ in which $\left|w_{1}(z, \bar{z})\right|^{2} / E^{4}>0$, and there exist isothermal coordinates $(u, v)$ and a frame field satisfying $(2.1)$. Then from (2.2) we have

$$
\Delta \log \left(\left|w_{1}(z, \bar{z})\right|^{2} / E^{4}\right)=-4 \Delta \log E=8 E K, \quad \Delta=\partial^{2} / \partial u^{2}+\partial^{2} / \partial v^{2},
$$

because $K$ is given by $K=-(1 / 2 E) \Delta \log E$. It follows from $K>0$ and (2.4) that the function $\tilde{w}_{1}=\log \left(\left|w_{1}(z, \bar{z})\right|^{2} / E^{4}\right)$ is a subharmonic function on $M$. Since $\tilde{w}_{1}$ takes the maximum $\log A$ at $p_{0} \in U$, it must be constant $\log A$ on $U$. Furthermore, since $M$ is connected, $\tilde{w}_{1}$ is constant on $M$. It follows from this fact and (2.4) that $K$ is identically zero on $M$, which contradicts $K>0$. Thus the function $w_{1}(z, \bar{z})=0$, so $F_{1}$ and $G_{1}$ are orthonormal vectors in $N_{1}(p)$. Hence, we have proved our assertion.

If $m=1$, that is, $\operatorname{dim} N_{1}(p) \neq 0$ at each point $p \in M$, then, by Lemma 1 , we choose a neighborhood $U$ of $p$ in which there exist isothermal coordinates $(u, v)$ and frame fields satisfying (2.1) and

$$
\begin{aligned}
& \omega_{13}=k_{1} \omega_{1}=\omega_{24}, \quad \omega_{1 \beta}=\omega_{2 \beta}=0, \\
& \omega_{23}=-k_{1} \omega_{2}=-\omega_{14}, \quad 4<\beta,
\end{aligned}
$$

where $k_{1}$ is a positive differentiable function on $M$. Using the structure equations, from (2.5) we have

$$
\omega_{34}=2 \omega_{12}-\left(\log k_{1}\right)_{2} \omega_{1}+\left(\log k_{1}\right)_{1} \omega_{2},
$$

where $d\left(\log k_{1}\right)=\sum_{j}\left(\log k_{1}\right)_{j} \omega_{j}$. Furthermore, by (2.5) we may put

$$
k_{1} \omega_{3 \beta}=f_{\beta} \omega_{1}+g_{\beta} \omega_{2}, \quad k_{1} \omega_{4 \beta}=g_{\beta} \omega_{1}-f_{\beta} \omega_{2}, \quad 4<\beta,
$$

and define two normal vectors $F_{2}=\sum f_{\beta} e_{\beta}$ and $G_{2}=\sum g_{\beta} e_{\beta}$ on $U$. Then, from $\varphi_{2}$ we have the mapping $\tilde{\varphi}_{2}$ from $M_{p}$ into $N_{2}(p)$ as

$$
\tilde{\varphi}_{2}(X):=\varphi_{3}(X, X, X)=\cos 3 \theta \cdot F_{2}+\sin 3 \theta \cdot G_{2},
$$

for $X=\cos \theta \cdot e_{1}+\sin \theta \cdot e_{2} \in M_{p}, p \in U$. Using the structure equations, by (2.5) and (2.7) the complex valued function

$$
w_{2}(z, \bar{z})=E^{3}\left(\left|G_{2}\right|^{2}-\left|F_{2}\right|^{2}\right)+2 i E^{3}\left\langle F_{2}, G_{2}\right\rangle
$$

is holomorphic in $z$. Furthermore, $\left|w_{2}(z, \bar{z})\right|^{2} / E^{6}$ is a differentiable function on $M$. Hence, in the same way as Lemma 1 , we can prove the following 
LEMMA 2. If $m=1$, then, at each point $p \in M$, the image of a unit tangent circle $S_{p}^{1}$ under $\tilde{\varphi}_{2}$ is a point or a circle according as $\operatorname{dim} N_{2}(p)-\operatorname{dim} N_{1}(p)=0$ or $\neq 0$.

If $m=2$, that is, $\operatorname{dim} N_{2}(p)-\operatorname{dim} N_{1}(p)>0$ at each point $p \in M$, then, by Lemma 1 and Lemma 2, we choose a neighborhood $U$ of $p$ in which there exist isothermal coordinates $(u, v)$ and frame fields satisfying $(2.1),(2.5)$ and

$$
\begin{aligned}
& k_{1} \omega_{35}=k_{2} \omega_{1}=k_{1} \omega_{46}, \quad \omega_{3 \gamma}=\omega_{4 \gamma}=0, \\
& k_{1} \omega_{36}=k_{2} \omega_{2}=-k_{1} \omega_{45}, \quad 6<\gamma,
\end{aligned}
$$

where $k_{2}$ is a positive differentiable function on $M$. Let $d\left(\log k_{2}\right)=\sum\left(\log k_{2}\right)_{j} \omega_{j}$; then from (2.9) we have

$$
\omega_{56}=3 \omega_{12}-\left(\log k_{2}\right)_{2} \omega_{1}+\left(\log k_{2}\right)_{1} \omega_{2}
$$

and we may write

$$
k_{2} \omega_{5 \gamma}=f_{\gamma} \omega_{1}+g_{\gamma} \omega_{2}, \quad k_{2} \omega_{6 \gamma}=g_{\gamma} \omega_{1}-f_{\gamma} \omega_{2}, \quad 6<\gamma .
$$

From the third shape operator $\varphi_{3}$ we have the mapping $\tilde{\varphi}_{3}$ from $M_{p}$ into $N_{3}(p)$ as

$$
\tilde{\varphi}_{3}(X):=\varphi_{3}(X, X, X)=\cos 4 \theta \cdot F_{3}+\sin 4 \theta \cdot G_{3},
$$

for $X=\cos \theta \cdot e_{1}+\sin \theta \cdot e_{2} \in M_{p}, p \in M$, where $F_{3}=\sum f_{\gamma} e_{\gamma}$ and $G_{3}=\sum g_{\gamma} e_{\gamma}$ are normal vector fields on $U$. Furthermore, from (2.9), (2.10) and (2.11) we see that the complex function

$$
w_{3}(z, \bar{z})=E^{4}\left(\left|G_{3}\right|^{2}-\left|F_{3}\right|^{2}\right)+2 i E^{4}\left\langle F_{3}, G_{3}\right\rangle
$$

is holomorphic in $z$.

Continuing this way, we have the following:

THEOREM 1. Let $M$ be a connected compact oriented surface of positive curvature minimally immersed in a $(2+\nu)$-dimensional space form $\tilde{M}$ of constant curvature $c$. If the immersion is $m$-regular, then, at each point on $M$, the image of a unit tangent circle $S_{p}^{1}$ under the mapping $\tilde{\varphi}_{n}$ (the nth shape operator) is a circle for any $n=1,2, \ldots, m$, and the image of $S_{p}^{1}$ under $\tilde{\varphi}_{m+1}$ is a point or a circle according as $\operatorname{dim} N_{m+1}(p)-\operatorname{dim} N_{m}(p)=0$ or $\neq 0$.

PROOF. For $n=1,2$, we have proved our assertion in Lemma 1 and Lemma 2. By induction on $n$, we will prove our assertion. So we assume that the above assertion holds for all $t \leq n-1<m, 3 \leq n$. Then we can choose a neighborhood $U$ of a point $p \in M$ in which there exist isothermal coordinates $(u, v)$ and frame fields satisfying (2.1) and

$$
\left\{\begin{array}{l}
k_{t-1} \omega_{\alpha_{1} \beta_{1}}=k_{t} \omega_{1}=k_{t-1} \omega_{\alpha_{2} \beta_{2}}, \quad \omega_{\alpha_{1} \gamma}=\omega_{\alpha_{2} \gamma}=0 \\
k_{t-1} \omega_{\alpha_{1} \beta_{2}}=k_{t} \omega_{2}=-k_{t-1} \omega_{\alpha_{2} \beta_{1}}, \quad 2 t+2<\gamma \\
\alpha_{1}=2 t-1, \quad \alpha_{2}=2 t, \quad \beta_{1}=2 t+1, \quad \beta_{2}=2 t+2, \\
t=1,2, \ldots, n-1,
\end{array}\right.
$$

where $k_{0}=1$ and $k_{t}(1 \leq t \leq n-1)$ are positive differentiable functions on $M$. Using the structure equations, from $(2.12)_{n-1}$ we have

$$
\omega_{\beta_{1} \beta_{2}}=(t+1) \omega_{12}-\left(\log k_{t}\right)_{2} \omega_{1}+\left(\log k_{t}\right)_{1} \omega_{2},
$$


where $\beta_{1}=2 t+1, \beta_{2}=2 t+2$, and $d\left(\log k_{t}\right)=\sum\left(\log k_{t}\right)_{j} \omega_{j}$ for $t=1,2, \ldots, n-1$. Furthermore, from $(2.12)_{n-1}$ we may write

$$
\begin{gathered}
k_{n-1} \omega_{a_{1} \gamma}=f_{\gamma} \omega_{1}+g_{\gamma} \omega_{2}, \quad a_{1}=2 n-1, \\
k_{n-1} \omega_{a_{2 \gamma}}=g_{\gamma} \omega_{1}-f_{\gamma} \omega_{2}, \quad a_{2}=2 n, \quad 2 n<\gamma .
\end{gathered}
$$

From the $n$th shape operator $\varphi_{n}$ we have the mapping $\tilde{\varphi}_{n}$ from $M_{p}$ into $N_{n}(p)$ as $\tilde{\varphi}_{n}(X):=\tilde{\varphi}_{n}(X, \ldots, X)$ for $X \in M_{p}$. For a unit tangent vector $X=\cos \theta \cdot e_{1}+$ $\sin \theta \cdot e_{2}$ at $p$ to $M$, we have

$$
\tilde{\varphi}_{n}(X)=\cos (n+1) \theta \cdot F_{n}+\sin (n+1) \theta \cdot G_{n},
$$

where $F_{n}=\sum f_{\gamma} e_{\gamma}$ and $G_{n}=\sum g_{\gamma} e_{\gamma}$ are normal vector fields on $U$. Using the structure equations, from $(2.12)_{n-1}$ and $(2.13)_{n-1}$ we see that the complex valued function

$$
w_{n}(z, \bar{z})=E^{n+1}\left(\left|G_{n}\right|^{2}-\left|F_{n}\right|^{2}\right)+2 i E^{n+1}\left\langle G_{n}, F_{n}\right\rangle
$$

is holomorphic in $z=u+i v$. As stated above, we easily see that

$$
\left|w_{n}(z, \bar{z})\right|^{2} / E^{2 n+2}=\left\{\left|G_{n}\right|^{2}-\left|F_{n}\right|^{2}\right\}^{2}+4\left\langle G_{n}, F_{n}\right\rangle^{2}
$$

is a differentiable function on $M$. Hence, in the same way as in the proof of Lemma 1 , at each point $p \in M$, the image of $S_{p}^{1}$ under $\tilde{\varphi}_{n}$ is a circle, because $n \leq m$. Furthermore, when $n=m+1$, by the above consideration we see that the image of $S_{p}^{1}$ under $\tilde{\varphi}_{m+1}$ is a point or a circle according as $\operatorname{dim} N_{m+1}(p)-\operatorname{dim} N_{m}(p)=0$ or $\neq 0$. Thus we have proved our assertion.

Now we can prove the following:

THEOREM 2. Let $M$ be a connected compact oriented surface minimally immersed in $a(2+\nu)$-dimensional space form $\tilde{M}$ of constant curvature $c$. If the immersion is $m$-regular and the Gaussian curvature $K$ satisfies the inequality

$$
\frac{2 c}{(m+2)(m+3)} \leq K \leq \frac{2 c}{(m+1)(m+2)},
$$

then $M$ is of constant curvature $2 c /((m+2)(m+3))$ or $2 c /((m+1)(m+2))$.

PROOF. First we consider the case that $\operatorname{dim} N_{m+1}(p)=\operatorname{dim} N_{m}(p)$ at each point $p \in M$, so $k_{m+1}^{2}=0$ on $M$ by Theorem 1 . Using the structure equations, from (2.5), (2.12) and (2.13) we have

$$
\begin{aligned}
\Delta \log k_{t} & =E\left\{(t+1) K-2 k_{t}^{2} / k_{t-1}^{2}+2 k_{t+1}^{2} / k_{t}^{2}\right\}, \quad t=1,2, \ldots, m-1, \\
\Delta \log k_{m} & =E\left\{(m+1) K-2 k_{m}^{2} / k_{m-1}^{2}\right\}, \quad \Delta=\partial^{2} / \partial u^{2}+\partial^{2} / \partial v^{2},
\end{aligned}
$$

which imply

$$
\Delta \log \left(k_{1} \cdot k_{2} \cdots k_{m}\right)=E\left\{\frac{m(m+3)}{2} K-2 k_{1}^{2}\right\}=E\left\{\frac{(m+1)(m+2)}{2} K-c\right\}
$$

because $K=c-2 k_{1}^{2}$. Since $K \leq 2 c /((m+1)(m+2))$, (2.16) implies that the function $\log \left(k_{1} \cdots k_{m}\right)$ is a superharmonic function on $M$. Since $M$ is compact, $\log \left(k_{1} \cdots k_{m}\right)$ must be constant on $M$, which, together with (2.16), implies $K=$ $2 c /((m+1)(m+2))$ identically on $M$. 
Next, we consider the case that there exists a point $p_{0} \in M$ such that $\operatorname{dim} N_{m+1}\left(p_{0}\right)>\operatorname{dim} N_{m}\left(p_{0}\right)$. We choose a neighborhood $U$ of $p_{0}$ in which there exist isothermal coordinates $(u, v)$ and frame fields satisfying $(2.1)$ and $(2.12)_{m+1}$. We must remark that $k_{m+1}^{2}$ is a differentiable function on $M$ and vanishes at a point where $\operatorname{dim} N_{m+1}(p)=\operatorname{dim} N_{m}(p)$. On $U$ we can consider the image of $S_{p}^{1}$ under the mapping $\tilde{\varphi}_{m+2}$. For the same reason as above, it is a point or a circle according as $\operatorname{dim} N_{m+2}(p)-\operatorname{dim} N_{m+1}(p)=0$ or $\neq 0$. Using the structure equations, from (2.5), $(2.12)_{m+1}$ and $(2.13)_{m+1}$ we have

$$
\Delta \log k_{t}=E\left\{(t+1) K-2 k_{t}^{2} / k_{t-1}^{2}+2 k_{t+1}^{2} / k_{t}^{2}\right\}, \quad t=1, \ldots, m+1,
$$

where $k_{m+2}^{2}=\sum_{2(m+2)<\gamma} f_{\gamma}^{2}$ is the square of the radius of the image of $S_{p}^{1}$. From (2.16) we have

$$
\begin{aligned}
\Delta\left(\log k_{1} \cdots k_{m+1}\right) & =E\left\{\frac{(m+1)(m+4)}{2} K-2 k_{1}^{2}+2 k_{m+2}^{2} / k_{m+1}^{2}\right\} \\
& \geq E\left\{\frac{(m+2)(m+3)}{2} K-c\right\},
\end{aligned}
$$

which, together with $K \geq 2 c /((m+2)(m+3))$, implies $\log \left(k_{1} \cdots k_{m+1}\right)$ is a subharmonic function on $U$. Here we may assume that the differentiable function $k_{1}^{2} \cdots k_{m+1}^{2}$ takes the maximum value at $p_{0}$. Then $\log \left(k_{1} \cdots k_{m+1}\right)$ takes its maximum at $p_{0}$ in $U$, so it must be constant on $U$. Hence (2.18) implies that $K=2 c /((m+2)(m+3))$ on $U$. Since $M$ is connected, $K=2 c /((m+2)(m+3))$ on $M$. Thus we have proved our assertion.

We see that our main theorem is obtained as a corollary of the results in [3] and the above Theorem 2 .

\section{REFERENCES}

1. E. Calabi, Minimal immersions of surfaces in euclidean spheres, J. Differential Geom. 1 (1967), 111-125.

2. M. P. do Carmo and N. R. Wallach, Minimal immersions of spheres into spheres, AMS, 93 (1971), 43-62.

3. T. Itoh, Minimal surfaces in a Riemannian manifold of constant curvature, Kōdai Math. Sem. Rep. 25 (1973), 202-214.

4. __ On minimal surfaces in a Riemannian manifold of constant curvature, Math. J. Okayama Univ. 17 (1974), 19-38.

5. T. Otsuki, Minimal submanifolds with $M$-index 2 and generalized Veronese surfaces, J. Math. Soc. Japan 24 (1972), 89-122.

INSTitute of Mathematics, UNiVersity of TSUKUba, TSUKUba-Shi, Ibaraki, 305 JAPAN 